\title{
Treatment with ADT impairs cognitive performance
}

Findings of a new study, published in the Journal of Clinical Oncology, demonstrate that treatment with androgen deprivation therapy (ADT) results in a decline in cognitive performance in patients with prostate cancer.

"There has been some debate in the field about whether ADT, which is commonly prescribed for prostate cancer, can worsen patients' cognitive functioning," explains lead author Brian Gonzalez, who adds "contradictory findings have been reported, but these studies were largely cross-sectional (as opposed to longitudinal), had short follow-up periods, or focused relatively little on clinically significant changes in cognitive function."

In order to more conclusively address this question, patients receiving ADT underwent assessments of cognitive function before or within 21 days of commencing ADT. These patients then underwent further assessments after 6 months or 12 months of treatment, and results of cognitive function tests were compared with those of age-matched and education-matched patients who were treated with prostatectomy alone, or did not have any history of prostate cancer.

Cognitive performance between groups was similar at baseline; however, after 6 months or 12 months, patients who received ADT had an increased risk of impaired cognitive performance of approximately 1.7-fold after 6 months and more than twofold after 12 months.

\section{$\mathbf{4}$...ADT can worsen cognitive function in patients with prostate cancer... 77}

Investigators also looked at genetic predictors of impaired performance. A total of 353 single-nucleotide polymorphism were investigated, of which only one, rs104776 in the guanine nucleotide binding protein (G protein), beta polypeptide 3 (GNB3) gene, conformed to all selection criteria.
Patients with two G alleles of rs 1047776 were 14 times more likely to have impaired cognitive performance compared with all other patients, including those who were homozygous for this polymorphism. These findings have important implications for management of patients receiving ADT.

"The finding of worse cognitive impairment over time helps provide evidence for the idea that ADT can worsen cognitive function in patients with prostate cancer," concludes Gonzalez. Furthermore, describing the clinical relevance of the genetic findings, Gonzalez adds "these results help lead us to a future when we can carefully tailor cancer treatment to the unique needs of each patient."

\section{Peter Sidaway}

Original article Gonzalez, B. D. et al. Course and predictors of cognitive function in patients with prostate cancer receiving androgen-deprivation therapy: a controlled comparison. J. Clin. Oncol. doi:10.1200/ JC0.2014.60.1963 